\title{
A Study on the Synchronization Characteristics of Thermoacoustic Laser
}

\author{
Sung Seek Park, Seung Jin Oh, Won Gee Chun, Kuan Chen, and Nam Jin Kim
}

\begin{abstract}
Thermoacoustic lasers convert heat from a high temperature heat source into acoustic power while rejecting waste heat to a low temperature sink. Recent research mainly focuses on using thermoacoustic engine arrays. Therefore, this study mainly focuses on coupling two thermoacoustic lasers. The coupling between the two lasers was started at $0^{\circ}$ crossing angle, where the openings of the lasers were parallel to each other and separated by a distance of $1 \mathrm{~m}$. The next configuration was to focus the sound waves using two different crossing angles $\left(30^{\circ} \& 90^{\circ}\right)$ between the two lasers, with their opening ends placed very close to each other. As a result, we conclude from all the measurements made for both the $30^{\circ}$ and $90^{\circ}$ crossing experiments that synchronization between two lasers is possible when their open ends were placed close to each other.
\end{abstract}

Index Terms-Thermoacoustic laser, convert, crossing angle, synchronization, sound pressure level.

\section{INTRODUCTION}

Thermoacoustics is a field that involves the study of both acoustics and thermodynamics. In other words it is known as the study that involves sound waves and the conversion of one form of energy into another. When a sound wave travels through a gas, it creates pressure and velocity oscillations within the gas it travels. These oscillations will produce a "thermoacoustic" effect. The engines which produce "thermoacoustic" effects are known as thermoacoustic engines. Thermoacoustic engines find their use in various fields like medicine, thermodynamics, fluid mechanics and material sciences etc. For example, ultrasound is used to shatter kidney stones. Shock waves are used in mining and material processing. They are also used in cleaning and sterilizing surfaces and medical instruments by sanitizing liquids. Recent developments in thermoacoustic engines like using solar energy or waste heat to run them makes it promising and economically sound for generating large quantities of acoustic energy. These engines have been looked at with increased interest in recent years due to useful applications like converting thermal energy into acoustic energy (thermoacoustic heat engines) and acoustic energy into refrigeration (thermoacoustic refrigerator).

In the 1970s' P. Ceperley proposed and built the first traveling wave thermoacoustic engine [1]. Applying the

Manuscript received October 19, 2012; revised January 30, 2013.

Sung Seek Park, Seung Jin Oh, Won Gee Chun, and Nam Jin Kim are with the Department of Nuclear \& Energy Engineering, Jeju National University, Jeju, Korea (e-mail: pss5153@jejunu.ac.kr, osj2558@jejunu.ac.kr,wgchun@jejunu.ac.kr,jnkim@jejunu.ac.kr).

Kuan Chen is with the Department of Mechanical Engineering, University of Utah, Salt Lake City, USA (e-mail: chen@mech.utah.edu). principle of superposition, Fahey suggested placing the maximum heat gradient at the point of maximum gas molecule velocity [2]. In thermoacoustic devices, the maximum gas molecule velocity appears at approximately one third of the tube length from the closed end. Yong Tae Kim and Min Gon Kim studied stack position dependency of a thermoacoustic prime mover based on the temperature difference across the stack [3]. The authors measured acoustically produced temperature deference across the stack as the function of stack position and sound frequency. In a recent study, Ryan Lampe used Selective Laser Sintering to produce a gamut of stack arrays such as square tubes, parallel plates, and a pin array [4]. These fabricated stacks were then tested and their performances were compared using a compressor driven closed tube heat pump. It was observed that the drop of $4.3 \mathrm{C}$ can be obtained across a parallel plate stack using a $12-\mathrm{W}$ amplifier, with $130 \mathrm{mV}$ peak-to-peak of the sine-wave audio signal. Symko and his co-workers suggested the use of thermoacoustic prime movers for thermal management and electrical energy generation in the systems which are sources of heat [5], [6]. The quarter wavelength thermoacoustic prime movers developed by Symko generated sound waves of frequency up to $24 \mathrm{kHz}$. The sound generated by these prime movers was then converted to electricity using a $3 \mathrm{~cm}$ diameter piezoelectric material, PZT, in the unimorph configuration thus providing thermal management and electrical energy generation. Recent research mainly focuses on using thermoacoustic engine arrays, including "Coupling of mid-audio frequency thermoacoustic engines" and "A study of coupled thermoacoustic engines" by Symko et. al. [7], [8]. These studies focus on coupling an array of engines using both mass coupling and acoustic coupling techniques.

Therefore, the purpose of this study was to acoustically couple the two thermoacoustic lasers and study the interaction between the acoustic waves generated by the coupling. In some of the experiments, we focused the sound waves of two thermoacoustic lasers, in order to understand the effects of coupling and determine the acoustic amplitude of the coupled sound wave.

\section{EXPERIMENTAL APPARATUS}

Fig. 1 shows a schematic diagram of the experimental apparatus used in this study. The experimental apparatus consists of a Pyrex tube as a resonator, a Celcor ceramic catalytic converter as a stack material, copper magnet wire with enamel insulation, nichrome $(\mathrm{NiCr})$ resistance heater wire and DC power supply for making necessary electricity.

Also, at the open end of the laser, we mount one of two different devices: a sound pressure level meter, a miniature 
microphone. These measuring devices were used to observe and record sound pressure level, amplitude, frequency and phase difference in all the experiments conducted. The microphones act as an acoustic transducer.

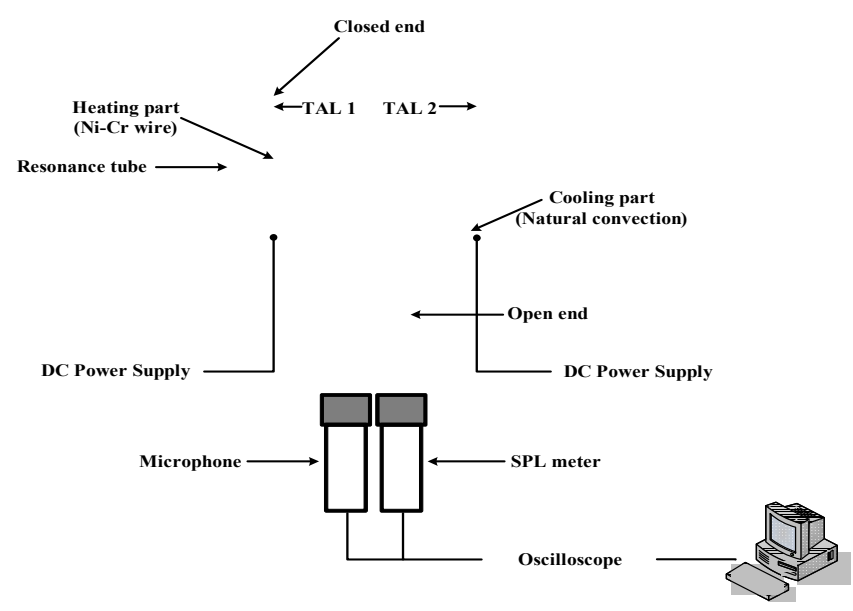

Fig. 1. Schematic diagram of Thermoacoustic laser system experimental apparatus (a)

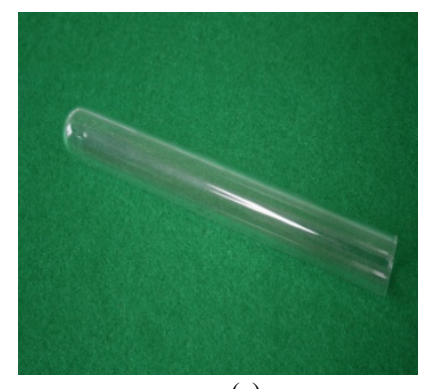

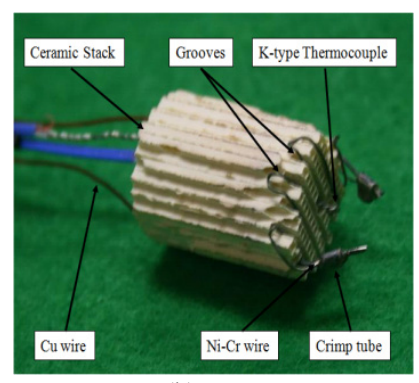

(b)
Fig. 2. Photographs of a resonance tube(a) and $\operatorname{stack(b)}$

The stack consisted of a K-type thermocouple, a crimp tube, a 26 gauge nichrome $(\mathrm{NiCr})$ resistance heater wire with $2.56 \mathrm{ohms}$ resistance per foot, and a 24 gauge copper magnet wire with enamel insulation. 6 evenly spaced horizontal grooves of $3 \mathrm{~mm}$ depth, were made on the front face of the stack, as shown in Fig. 2. The NiCr heater wire was adhered inside these groves. The ends of the $\mathrm{Cu}$ connecting wires were stripped by burning the enamel insulation. Two $\mathrm{Cu}$ wires were inserted through the channels of the stack and their stripped ends were twisted with $\mathrm{NiCr}$ wire to make good electrical contact. The stack was heated with the help of a nichrome $(\mathrm{NiCr})$ wire wound on one side of the stack and the other part was cooled by radiation and natural convection to the atmospheric air in the Pyrex tube. We used a data acquisition card to measure and record the amplitude, frequency and the phase difference of the signals read by both microphones. In particular, the National Instruments DAQ card (NI 6009) was connected to the microphones. The NI USB 6009 provides connection to eight analog input (AI) channels, two analog output (AO) channels, 12 digital input/output (DIO) channels, and a 32-bit counter with a Full-Speed USB interface.

\section{EXPERIMENTAL METHOD AND RESUlts}

\section{A. Single Termoacoustic Laser}

We conducted experiments to study the nature of the acoustic signal generated from a single thermoacoustic laser.
To study the nature of the spherical waves that exit the opening of the laser tube, we measured the sound intensity at various distances from the open end of the laser. Sound intensity was measured in decibels by using a sound pressure level meter oriented in three different styles: parallel, perpendicular and at a $45^{\circ}$ angle to the axis of the laser.

Fig. 3 depict the variations in sound pressure levels for the three different orientations and input power rates. The sound pressure level at the open end of the laser tube was about 120 $\mathrm{dB}$ for an electric power input of $25 \mathrm{~W}$. The sound pressure level decreased by $20 \mathrm{~dB}$ for each increment of $2 \mathrm{~cm}$, from $\mathrm{r}=$ $2 \mathrm{~cm}$ to $\mathrm{r}=20 \mathrm{~cm}$. This confirms that the sound pressure level of the sound waves generated outside the laser tube decreases with distance according to $1 / \mathrm{r}$ (given by the inverse distance law). In all three orientations, at any distance greater than 10 $\mathrm{cm}$ from the open end of the laser tube, the sound intensities were almost the same. At $\mathrm{r}=0.4 \mathrm{~m}$, there was a slight increase in pressure level. We conjecture that this could be due to either the reflected sound waves or phase differences between harmonics.

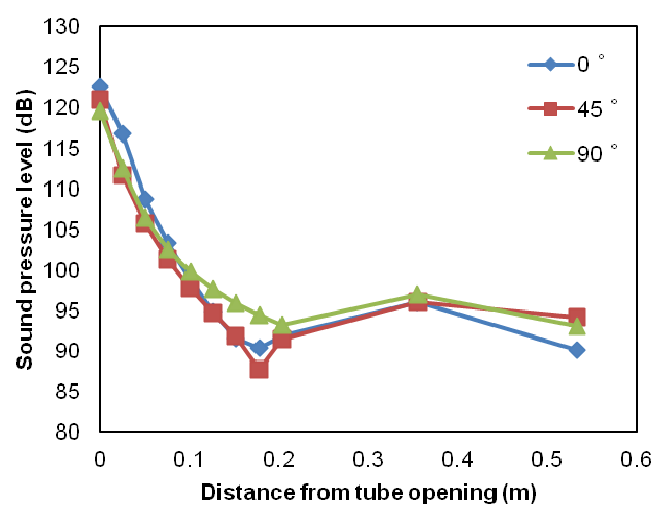

Fig. 3. Sound pressure levels measured in all three orientations $\left(0,45, \& 90^{\circ}\right)$ for high powered lasers.

\section{B. Zero Crossing Angle}

We designed this experiment to study the interaction between the sound waves by coupling the signals of two thermoacoustic lasers placed parallel to each other and separated by one meter distance (zero crossing angle). we used two microphones, M1 and M2, to measure and record frequency and phase.

Fig. 4 and Fig. 5 show the signals read by M1 and M2. The arrows in both Fig. 4 and Fig. 5 indicate the movement of the signal read using M2. This movement indicates the phase change of the signals periodically going from in-phase to out-of-phase. As shown in Fig. 5, at a particular time the two signals will couple with each other in such a way that causes constructive interference. In all the experimental runs, we did not observe the synchronization by acoustically coupling the signals of TA1 and TA2. This was mainly due to the spatial distance between the two lasers. When the lasers are far apart, they operate with their separate natural frequencies. This will cause the signals to "beat" at the difference frequency. The atmospheric air between the lasers also acts as resistance and it does not allow the signals to couple in a single phase, increasing the "detuning". When the separating distance between the two lasers is larger, the detuning is increased and the coupling becomes weaker. Therefore, in this particular case, synchronization or phase locking between the parallel 
lasers is not observed.

The amplitude increased slightly when the acoustically coupled signals of the two lasers were in-phase with each other, while it decreased when the signals were out-of-phase. We observed the same phenomenon with the third microphone. The arrows in Fig. 6 indicate that the amplitude of the signal recorded by the LM oscillates between its maximum and minimum values when the phase difference between the signals of the lasers TA 1 and TA 2 changed from 0 to 180 degree.

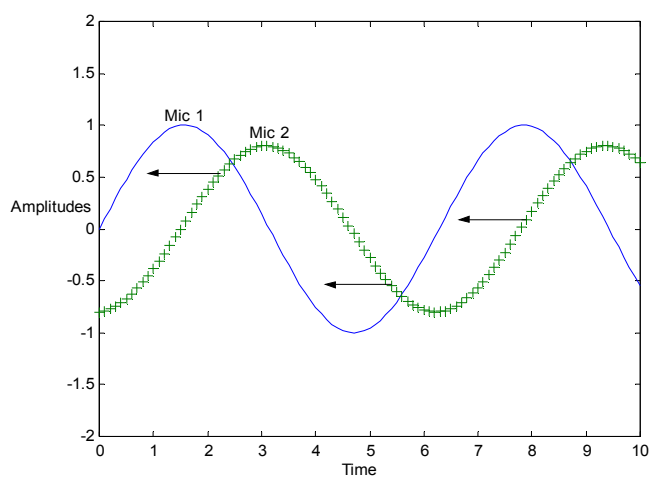

Fig. 4. Out-of-phase sound waves measured by M1 and M2 when two TA lasers were seperated by one meter.

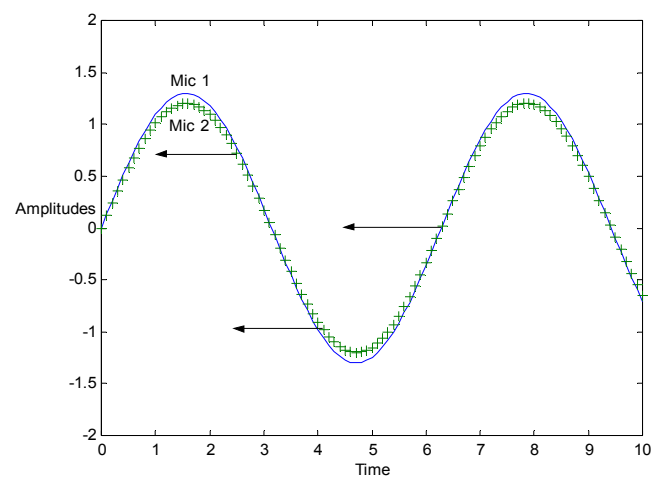

Fig. 5. In-phase sound waves measured by M1 and M2 when two TA lasers were seperated by one meter.

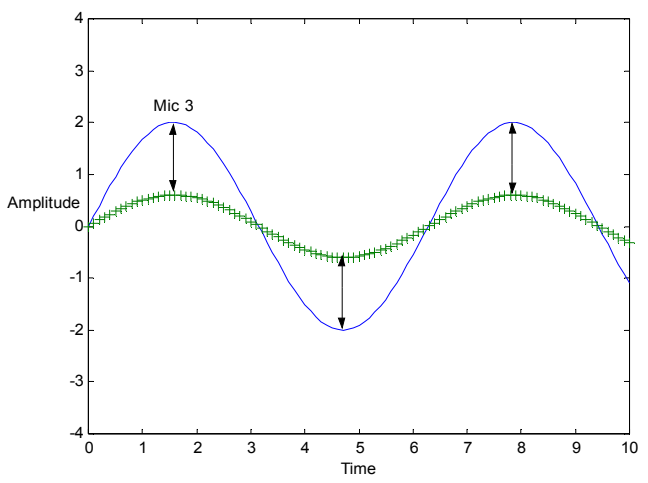

Fig. 6. The beat signal changing from in-phase to out-of-phase, measured by the LM (microphone 3)

\section{Small Crossing Angle $\left(30^{\circ}\right)$}

For the measurement of SPL at Crossing Angle $\left(30^{\circ}\right)$, a sound pressure level meter was used. It was placed at $20 \mathrm{~cm}$ away from the openings of the two lasers. In this case, the lasers had higher power ratings, with TA1 and TA2 running at 32.4 and $34.3 \mathrm{~W}$, respectively. We started by switching on TA1 and recorded a sound pressure level of $95 \mathrm{~dB}$. A few seconds later, while TA1 was still running, we switched on TA2. Initially, the signals were beating, increasing and decreasing the sound pressure level. When the lasers beat at the difference frequency, the highest sound pressure level recorded was approximately $100 \mathrm{~dB}$. This beating of the initial unlocked signals was only observed for the first few seconds of the 2 minute experimental run.

The sound pressure level for the locked signal kept decreasing until it reached a minimum steady value around $80 \mathrm{~dB}$. Fig. 7 shows this decrease in the sound pressure level when both lasers were running. This indicates that the phase difference between the two sound waves is close to 180 degrees.

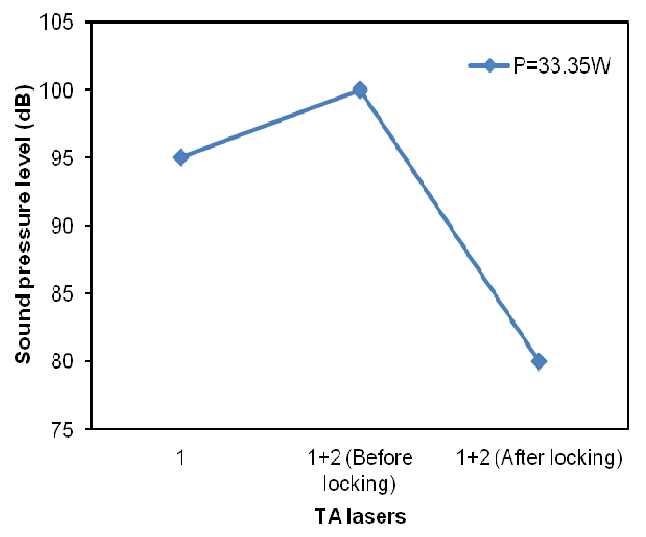

Fig. 7. Sound pressure levels recorded at $30^{\circ}$ crossing angle

Fig. 8 illustrates the signal recorded when both lasers TA1 and TA2 are running. In this experiment, we first switch on TA1 (powered with $32.4 \mathrm{~W}$ ) and later TA2 (powered with $34.3 \mathrm{~W}$ ). Synchronization was not observed immediately, because the signals do not lock in frequency and phase. The signals beat for the first 15 seconds of the 2 minute experiment run. In order to understand the nature of this beating signal wave form, the time interval is reduced to 0.240 seconds, as shown in Fig. 9. After running both lasers TA1 and TA2 for 15 seconds, the beat frequency slows and then abruptly stops, at which point the two lasers lock in a particular frequency and phase. Once synchronization was achieved, the signal amplitude decreased gradually as shown in the latter half of Fig. 8. To observe the nature of the synchronized wave form, the time interval is reduced to 0.240 seconds, as shown in Fig. 10. The amplitude recorded for the synchronized signal when both lasers were operating was smaller than the amplitude recorded when only laser TA1 was operating. This implies anti-phase synchronization.

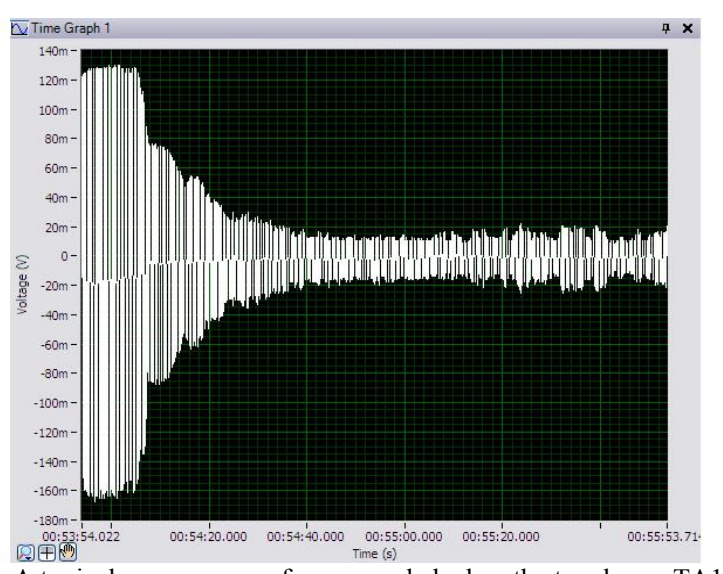

Fig. 8. A typical pressure waveform recorded when the two lasers TA1 and TA2 are crossing each other at $30^{\circ}$ (TA1 is switched on before TA2). 


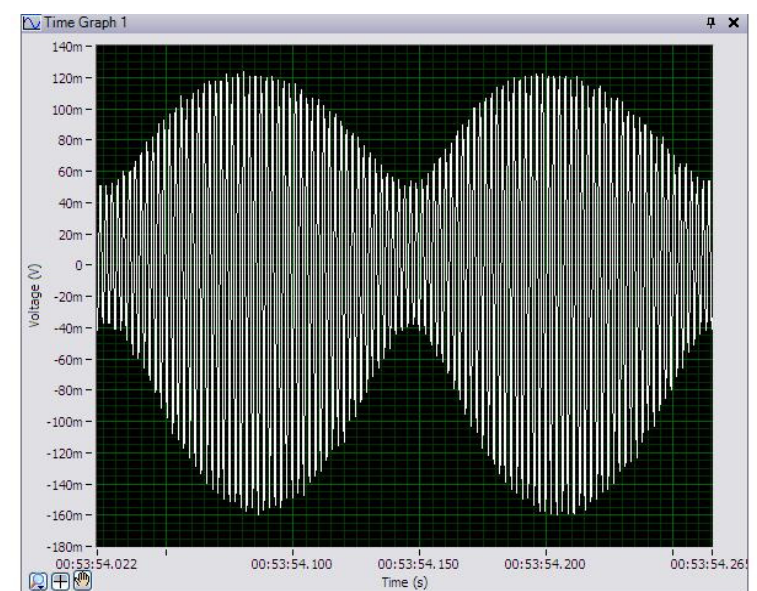

Fig. 9. A typical pressure waveform of the unlocked beat signal recorded when the two lasers TA1 and TA2 are crossing each other at $30^{\circ}$ (TA1 is switched on before TA2).

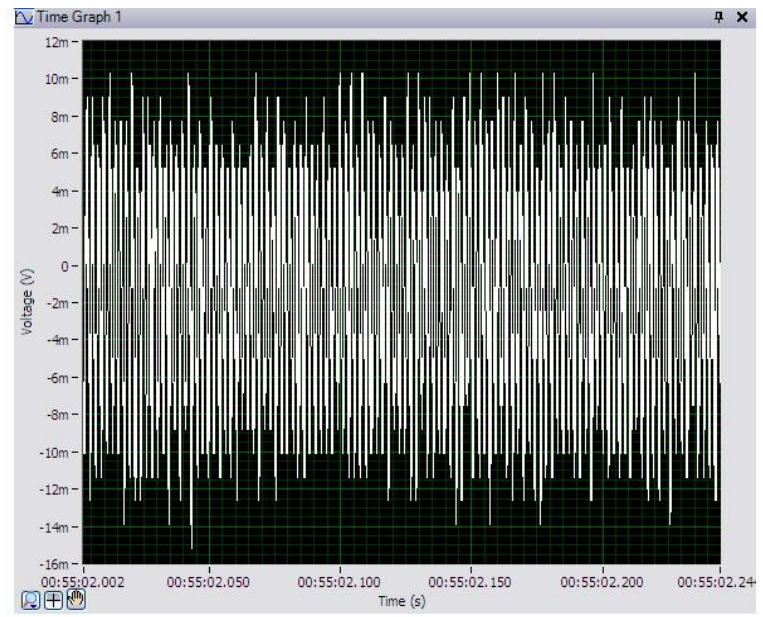

Fig. 10. A typical pressure waveform of the locked (synchronized) signal recorded when the two lasers TA1 and TA2 are crossing each other at $30^{\circ}$ (TA1 is switched on before TA2).

\section{A. Large Zero Crossing Angle $\left(90^{\circ}\right)$}

We designed this experiment to study the interaction between the sound waves of two thermoacoustic lasers when their openings are very close to each other and the angle between their longitudinal axes is $90^{\circ}$. In this case, the duration of the run was 2 minutes and the sound pressure level meter was placed at $20 \mathrm{~cm}$ away from the openings of the two lasers to avoid reflections. The decrease in sound pressure level of the synchronized signal was recorded and is shown in Fig. 11. Therefore, we can conclude that when the lasers were powered at the higher rate, the sound wave frequencies alter each other to lock in frequency and phase.

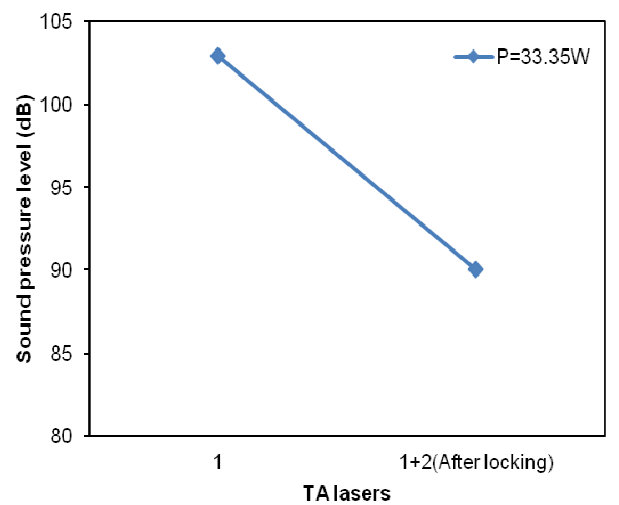

Fig. 11. Sound pressure levels recorded at $90^{\circ}$ crossing angle.
Fig. 12 illustrates the signal recorded when both lasers TA1 and TA2 are running. In this experiment, we first switch on TA1 (powered with $32.4 \mathrm{~W}$ ) and later TA2 (powered with $33.8 \mathrm{~W}$ ). Synchronization was observed immediately, and the signals lock in frequency and phase. To observe the nature of the synchronized wave form, the time interval is reduced to 0.240 seconds, as shown in Fig. 13. The immediate synchronization in this case is due to the close match in the initial frequencies of the two lasers. The amplitude recorded for the synchronized signal when both lasers were operating was smaller than the amplitude recorded when only laser TA1 was operating. This implies anti-phase synchronization.

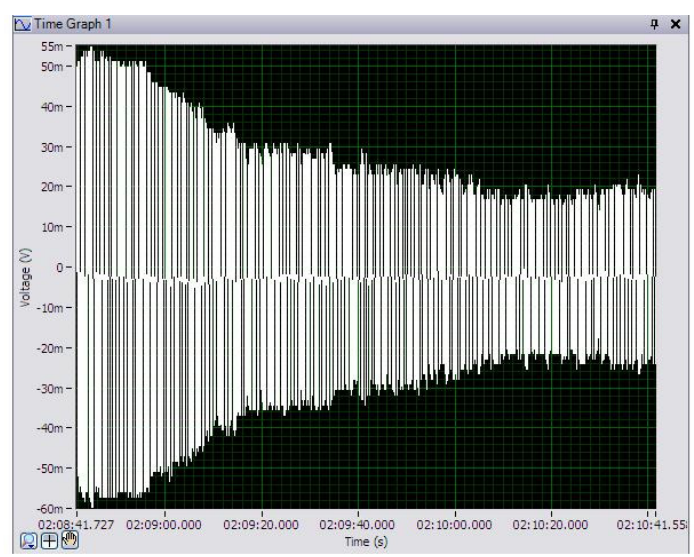

Fig. 12. A typical pressure waveform recorded when the two lasers TA1 and TA2 are crossing each other at $90^{\circ}$ (TA1 is switched on before TA2).

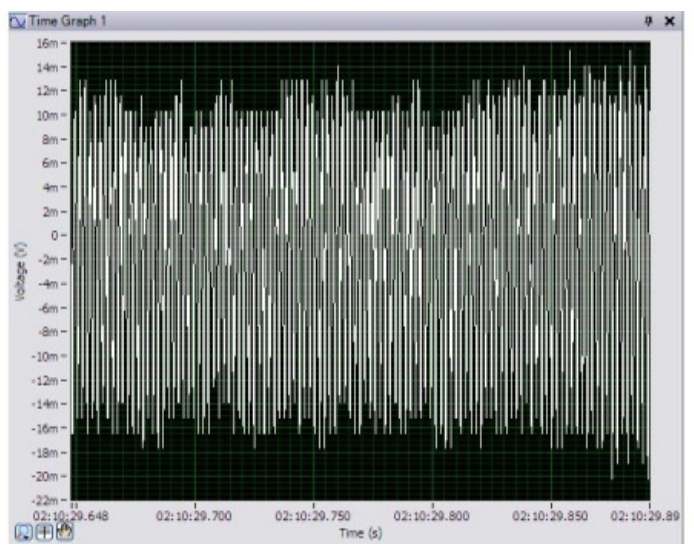

Fig. 13. A typical pressure waveform of the locked (synchronized) signal recorded when the two lasers TA 1 and TA2 are crossing each other at $90^{\circ}$ (TA1 is switched on before TA2).

\section{CONCLUSION}

The work presented in this thesis was to study the interaction between the sound waves, generated from the two thermoacoustic lasers which were coupled acoustically. Sound pressure levels were measured in three different orientations $\left(0,45\right.$, and $\left.90^{\circ}\right)$ from the open end of the tube, in order to study the nature of the spherical sound waves exiting the laser tube. For all the orientations, there was a slight variance in pressure levels in the close vicinity of the openend of the laser tube, whereas at distances greater than $10 \mathrm{~cm}$ away from the open end, the pressure levels were approximately equal. Coupling between the two lasers was very critical and influential. First coupling was done at $0^{\circ}$ crossing angle, where the lasers were parallel to each other and separated by a distance of $1 \mathrm{~m}$. In this particular case, the signals beat with the difference in the natural frequency. The 
frequency of each laser was different at onset and it was not possible to synchronize the two lasers with their mismatching frequencies.

Synchronization (mode-locking) was observed when the crossing angle was changed to 30 and $90^{\circ}$. In this case, the sound waves were focused with the opening ends of the laser tubes in close proximity. The output acoustic amplitude for the synchronized signal was always less compared to that of a single laser. We observed only out-of-phase synchronization for the two phase locked lasers. The synchronized locked signal was always out of phase, which resulted in decreased amplitude compared to that of a single laser. This decrease in amplitude for the locked signal is on an average comparatively $75 \%$ less than the amplitude of the single laser.

\section{ACKNOWLEDGEMENTS}

This work was supported by the National Research Foundation of Korea (NRF) grant funded by the Korea government(MEST) (No. 20120008672)

\section{REFERENCES}

[1] D. Fahey, "Thermoacoustic oscillations in cryogenics. Part 3: avoiding and damping of oscillations," Cryogenics, vol. 32, no. 8, pp.703-706, 1992.

[2] Y. T. Kim and M. G. Kim, "Optimum Positions of a Stack in a Thermoacoustic Heat Pump," Journal of the Korean Physical Society, vol. 36 , no. 5 , pp. $279-286,2000$.

[3] R. Lampe, "Design and Testing of Rapid Prototyped Stacks for Thermoacoustic Applications," in Proc. the National Conference on Undergraduate Research (NCUR), 2008, pp. 1-8.

[4] O. G. Symko, "Design and development of high-frequency thermoacoustic engines for thermal management in microelectronics," Microelectronics Journal, vol. 35, no. 2, pp. 185-191, 2004.

[5] O. G. Symko, "Acoustic approach to thermal management: miniature thermoacoustic engines," in Proc. Thermal and Thermomechanical 10th Intersociety Conference on Phenomena in Electronics Systems, ITHERM, 2006, pp. 771-776.

[6] P. H. Ceperley, "A pistonless Sturling engine- The travelling wave heat engine," Acoust. Soc. Am, vol. 66, no. 5, pp. 1508, 1979.

[7] B. G. McDonald and O.G. Symko, "Coupling of mid audio frequency thermoacoustic prime movers," Acoust. Soc. Am, vol. 125, no. 4, pp. 2563, 2009.

[8] B. J. Gillman, "A Study of Thermoacoustic Engines," University of Utah, 2009.

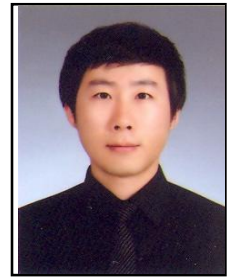

Sung Seek Park lives in 66 Jejudaehakno, Jejusi, Jejudo, Korea and date of birth 10.11. 1982. He graduated from Bachelor of engineering, Jeju national Uni.(2008), Master of engineering, Jeju national Uni.(2010). At present, he's a Ph. D candidate, Jeju national Uni. and the major is energy \& chemistry engineering. Typical paper is "A comparative study on the enhanced formation of methane hydrate using CM-95 and CM-100 MWCNTs". In. Com. In Heat and Mass Transfer, 2011.

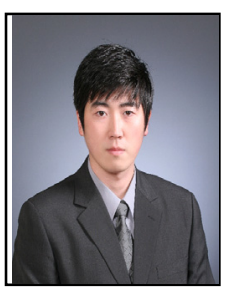

Seung Jin Oh lives in 66 Jejudaehakno, Jejusi, Jejudo, Korea and date of birth 06.16. 1980. He graduated from Bachelor of engineering, Jeju national Uni.(2005), Master of engineering, Jeju national Uni.(2008). At present, he's a $\mathrm{Ph}$. D candidate, Jeju national Uni. and the major is energy $\&$ chemistry engineering. Typical paper is "Acoustic waves generated by a TA (ThermoAcoustic) laser pair", Energy, 2012.

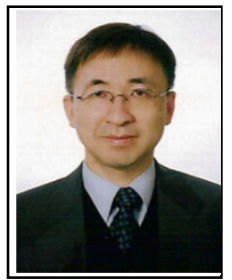

Won Gee Chun lives in 66 Jejudaehakno, Jejusi, Jejudo, Korea and date of birth 06.22. 1957. He graduated from Bachelor of engineering, Hanyang Uni.(1981), Master of engineering, Utah Uni.(1984) and : Doctor of engineering, Utah Uni.(1986). At present, he's a Professor in Jeju national Uni. and the major is heat engineering. Typical paper is "Acoustic waves generated by a TA (ThermoAcoustic) laser pair", Energy, 2012.

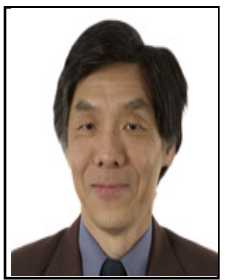

Kuan Chen lives in Salt Lake City, UT 84112, U.S.A and date of birth 03.01. 1953. He graduated from Bachelor of engineering, Chung Yuan Uni.91974), Master of engineering, Chung Yuan Uni.(1976), Doctor of engineering, Taiwan national Uni. And University of Illinois at Urbana- Champaign(1981). At present, he's a Professor in Utah Uni. and the major is mechanical engineering. Typical paper is "Acoustic waves generated by a TA (ThermoAcoustic) laser pair”, Energy, 2012.

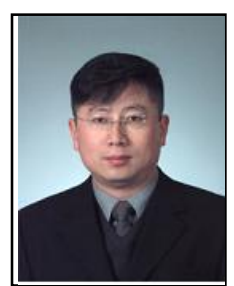

Nam Jin Kim live in 66 Jejudaehakno, Jejusi, Jejudo, Korea and date of birth 12. 01. 1967. He graduated from Bachelor of engineering, Inha Uni.(1993), Master of engineering, Inha Uni.(1995), Doctor of engineering, Inha Uni.(2003). At present, he's a Professor in Jeju national Uni. and the major is Heat fluid engineering. Typical paper is "Characteristic of methane hydrate formation in carbon nanofluids", J. of In. and Eng. Chem.,2012. 